\title{
Contributors
}

\section{Adam Auckburally}

BVSc CertVA DipECVAA PGCAP FHEA MRCVS Southern Counties Veterinary

Specialists, Unit 6 Forest Corner Farm, Hangersley, Ringwood,

Hampshire BH24 3JW

\section{Hazel Bentall}

BVSc MRCVS

Veterinary Intelligence Ltd,

12 Waverley Road, St Albans AL3 5PA

\section{John Chitty}

BVetMed CertZooMed CBiol MSB MRCVS

Anton Vets, Units 11-12 Anton Mill

Road, Andover, Hampshire SP10 2NJ

\section{Jonathan Cracknell}

BVMS CertVA CertZooMed MRCVS

Orchard Cottage, Knowsley Park,

Prescot L34 4AQ

\section{Giles Davis}

\section{BVSC GPCertSAP MRCVS}

Head of Legislation,

Veterinary Medicines Directorate,

Woodham Lane, New Haw, Addlestone, Surrey KT15 3LS

\section{Gillian Diesel}

\section{BVSc MSc PhD MRCVS}

Head of the Pharmacovigilance Team, Veterinary Medicines Directorate, Woodham Lane, New Haw, Addlestone, Surrey KT15 3LS

\section{Stephen Dawson}

MA

Secretary General, AMTRA, Unit 1c Woolpit Business Park,

Windmill Avenue, Bury St. Edmunds, Suffolk IP30 9UP

\section{Clive Elwood}

MA VetMB MSC PhD CertSAC DipACVIM-SAIM DipECVIM-CA FRCVS

The Old Schoolhouse,

23 Church Road, Little Berkhamsted, Hertfordshire SG13 8LY

\section{Sally Everitt}

BVSc MSc(VetGP) PhD MRCVS

Ross-on-Wye, Herefordshire

\section{Maggie Fisher}

BVetMed CBiol FRSB DipEVPC MRCVS

Veterinary Research Management,

The Mews Studio, Portland Road,

Malvern, Worcestershire WR14 2TA

\section{David Harris}

BVSC PGCert VetEd FHEA MRCVS

Lecturer in Veterinary Nursing,

Animal Health and Applied Veterinary

Pharmacology, Duchy College (and Bicton College)

\section{Jenny Helm}

BVMS BVMS CertSAM DipECVIM-CA FHEA MRCVS

School of Veterinary Medicine, University of Glasgow GL61 1QH

\section{Peter Holdsworth}

AO BSc(Hon) PhD FRSB FAICD

Shernacre Cottage,

Lower Howsell Road, Malvern, Worcestershire WR14 1UX

\section{Richard Jackson}

BMVS (Hons) MRCVS

St. David's Poultry Team,

Nutwell Estate, Lympstone,

Exmouth EX8 5AN

\section{Mike Jessop}

BVetMed MRCVS

Veterinary Expert, PO Box 575,

Swansea, SA8 9AW

\section{John Millward}

BSc(Hons) CBiol MRSB

Head of Compliance Unit, Veterinary Medicines Directorate, Woodham Lane, New Haw, Addlestone KT15 3LS

\section{Pam Mosedale}

BVetMed MRCVS

The Cottage, The Wash,

Chapel-en-le-Frith

Derbyshire SK23 OQW

\section{Donal Murphy}

MVB MRCVS

Head of Technical and Regulatory

Affairs, NOAH, 3 Crossfield Chambers, Gladbeck Way, Enfield EN2 7HF

\section{Fred Nind}

BVM\&S MRCVS

Harvest View Cottage,

Easter Breich,

West Lothian EH55 8PP

\section{Lea Reynolds}

Veterinary Medicines Directorate, Woodham Lane, New Haw, Surrey KT15 3LS

\section{Philip Sketchley}

BSc (Hons) Hon Assoc RCVS

Chairman AMTRA,

1c Windmill Avenue, Woolpit, Bury St Edmunds IP30 9UP

\section{Michael Stanford}

BVSC FRCVS

The Veterinary Defence Society

Limited, 4 Haig Court,

Parkgate Industrial Estate,

Knutsford, Cheshire WA16 8XZ

\section{Elizabeth Wright}

MPharm PgD

Royal (Dick) School of Veterinary

Studies, Easter Bush Campus,

Midlothian EH25 9RG 